\title{
Functional electrical stimulation (FES): muscle histochemical analysis
}

\author{
J M D Greve MD, R Muszkat PT, B Schmidt MD PhD, J Chiovatto MD,
} T E P Barros $\mathrm{F}^{\circ} \mathrm{MD}, \mathrm{L}$ R Batisttella MD

Department of Rehabilitation, Clinics Hospital, School of Medicine, University of São Paulo, São Paulo, Brazil.

Functional electrical stimulation (FES) has been used in Brazil since 1989 to obtain functional improvement in paraplegic patients' orthostasis and locomotion. The aim of this paper is to evaluate the histochemical changes observed in the quadriceps femoris muscle following the use of FES. We studied four patients with traumatic spinal cord lesions at T4-10 level, Frankel A, all within 12-24 months postlesion. They were all submitted to FES using the following criteria: square-wave, $20-30 \mathrm{~Hz}$ frequency, pulses of 0.003 seconds, time of stimulation 5 seconds, resting interval 10 seconds. The stimulation was applied during 90 consecutive days, 30 minutes each time, twice daily. The interval between the stimulations was 6 hours. Quadriceps muscle biopsies were performed before and after the use of FES. We used ATPase technique for the histochemical analysis, where three different dying patterns can be observed for the three types of muscular fibres (I, IIa and IIb). The two samples from each patient were analysed measuring the fibres' diameters and their index of atrophy, and counting the total number of each type of fibre in each sample. The mean total number of fibres in each sample was $256 \pm 12.3$. The results showed that the sizes of the three types of fibres were not modified with the use of FES; the number of type IIa fibres increased in a significant fashion, after using of FES.

Keywords: functional electrical stimulation; muscle histochemical; paraplegia; rehabilitation; spinal cord injury.

\section{Introduction}

Many European and North American centres have used FES for some years to promote locomotion in spastic paraplegic patients. This technique was introduced in Brazil in 1989. The option utilised here is the system developed by Gracanin ${ }^{1}$ which uses four channels of stimulation, applying surface electrodes to the quadriceps muscle and the fibular nerve.

Although the initial clinical responses to the treatment with FES were encouraging, with the possibility of some patients obtaining the upright position along with reciprocal locomotion, we thought that a more careful analysis of the muscle responses to the equipment used was necessary.

We therefore proposed an investigation of the muscular changes caused by FES by means of muscular biopsies and histochemical analysis, before treatment was started and after it had been carried out.

The human muscle is constituted of muscle fibres of variable enzymatic activity: type I fibres (red) that have high oxidative and low glycolitic activity and type II fibres (white) a high glycolitic and a low oxidative metabolism. Intermediate type fibres are also described in human muscle with different degrees of enzymatic oxidative and glycolitic activity. ${ }^{2}$

The inactive muscle, with lower motor neuron innervation preserved, shows decreased glycolitic and oxidative enzymatic activities, but type II fibres (white) undergo more atrophy when compared to type I fibres. ${ }^{2}$ Phasic stimulation leads to increased glycolitic activity, whilst tonic stimulation 
increases oxidative activity, implying that the kind of nervous stimulus is one of the factors that influences muscular metabolism. ${ }^{2}$

The aim of this work is to study the histochemical muscular changes of the quadriceps, when using FES in paraplegic patients with spinal cord lesions.

\section{Methods}

We chose four male patients who had traumatic spinal cord spastic lesions, at the thoracic level, Frankel A, with indications for FES in order to promote reciprocal locomotion and orthostasis. The details of the procedure, the methodology and any possible risks involved were explained to the patients. All four patients agreed to cooperate (Table I).

The selected patients were submitted to the FES programme according to the following criteria: square wave-form, impulse frequencies varying from 20 to $30 \mathrm{~Hz}$, impulses lasting 3 milliseconds, time of stimulation 5 seconds and 10 seconds of resting interval. The regions stimulated were the quadriceps femoris muscle with electrodes applied to the anterior-superior region of the thigh and the suprapatellar region. The electrodes were located at the spot for the best response, near the femoral nerve emergence in the thigh, in order to stimulate all the muscle fibres of the quadriceps. A superficial electrode was used and the patient was taught to put the electrode on the same place each time. In order to be sure of the correct place for the electrode, a skin mark was made. The electrodes employed were made of plastic material, size $4 \mathrm{~cm}^{2}$. The stand-up capacity of the patient with FES was used to ensure the efficiency of the

Table I Patients

\begin{tabular}{lccl}
\hline Patient & $\begin{array}{c}\text { Age } \\
\text { (years) }\end{array}$ & $\begin{array}{c}\text { Time since } \\
\text { lesion (months) }\end{array}$ & Level \\
\hline 1 & 31 & 15 & T10 \\
2 & 29 & 24 & T5 \\
3 & 28 & 12 & T10 \\
4 & 47 & 18 & T4 \\
\hline
\end{tabular}

quadriceps' electrically-induced contraction. The visual control was also used to improve the contraction of the vastus lateralis. The fibular nerve was also stimulated in order to obtain triple flexion and dorsal flexion of the foot.

During the home stay, the treatment was done twice daily, 30 minutes for each muscle (1 hour per muscle per day), 7 days per week, for 90 consecutive days. The interval between the stimulations was 6 hours. The quadriceps stimulation was done with the patient seated, so that the legs moved against gravity.

The equipment used was manufactured in Brazil, and consisted of a Kroman portable model for training, with two channels for stimulation. The equipment was gauged in the centre for rehabilitation and checked weekly throughout the period of investigation.

Biopsies of the vastus lateralis of the quadriceps muscle were performed before the initiation of the FES programme and 90 days after its completion. They were performed under aseptic conditions using a local anaesthetic. Through a $2 \mathrm{~cm}$ incision fragments of 3-4 mm were removed. The second biopsy was performed in the same limb, in the vastus lateralis, in a region close to the previous one. The muscle fragments were preserved in liquid nitrogen until the histochemical analysis submission date was due. We used the technique of ATPase miofibrilar activity analysis. ${ }^{3}$

Histochemical analysis was carried out by counting the number of fibres in each sample. We counted 200-500 fibres per sample (mean of $256 \pm 12.3$ fibres). The fibres were grouped according to the degree of (coloration) dye-uptake for each technique used; three groups were observed: type I, type IIa and type IIb fibres. The diameter of each fibre was measured and the average and standard deviation of all fibre types were calculated, in micra.

\section{Results}

1 No significant change was observed in the diameters of the fibres after using FES (Tables II, III, IV). As can be observed, the sizes of the fibres I, IIa and IIb did not 
Table II Size of the fibres type I (micra) before and after the use of FES

\begin{tabular}{lrr}
\hline Patient & \multicolumn{1}{c}{ Before } & \multicolumn{1}{c}{ After } \\
& \multicolumn{2}{c}{ (micra) } \\
\hline 1 & $25.24 \pm 8.84$ & $23.51 \pm 7.53$ \\
2 & $15.75 \pm 3.10$ & $25.19 \pm 6.72$ \\
3 & $28.5 \pm 11.25$ & $17.50 \pm 4.50$ \\
4 & $13.54 \pm 4.47$ & $13.50 \pm 2.25$ \\
\hline
\end{tabular}

Student's $t$-test: $p>0.05$

Table III Size of the fibres IIb (micra) before and after FES

\begin{tabular}{lrrr}
\hline Patient & \multicolumn{1}{c}{ Before } & \multicolumn{1}{c}{ After } \\
& \multicolumn{2}{c}{ (micra) } \\
\hline 1 & $18.47 \pm 7.20$ & $19.90 \pm 7.73$ \\
2 & $20.36 \pm 1.12$ & $28.21 \pm 13.72$ \\
3 & $30.50 \pm 12.00$ & $12.50 \pm 0.60$ \\
4 & $18.57 \pm 4.42$ & $12.25 \pm 3.00$ \\
\hline
\end{tabular}

Student's $t$-test: $p>0.05$

Table IV Size of the fibres IIa (micra) before and after use of FES

\begin{tabular}{lcc}
\hline Patient & Before & After \\
& \multicolumn{2}{c}{ (micra) } \\
\hline 1 & $21.70 \pm 5.97$ & $23.37 \pm 6.20$ \\
2 & $19.26 \pm 4.50$ & $31.81 \pm 11.48$ \\
3 & $25.75 \pm 5.19$ & $27.50 \pm 1.20$ \\
4 & $20.25 \pm 3.90$ & $14.00 \pm 4.50$
\end{tabular}

Student's $t$-test: $p>0.05$

show any difference in the averages obtained before and after 3 months of FES.

2 The number of type I fibres increased in two patients ( 2 and 3 ) and decreased in two cases ( 1 and 4$)$. These modifications were not statistically significant when evaluated by the chi-squared test (Fig 1).

3 The number of type IIb fibres decreased in three of the four patients $(2,3$ and 4$)$ and was constant in one (1). The decrease of type IIb fibres was significant in the sample studied (Fig 2).

4 The number of type IIa fibres increased in a significant fashion with the use of FES in all the samples studied (Fig 3).

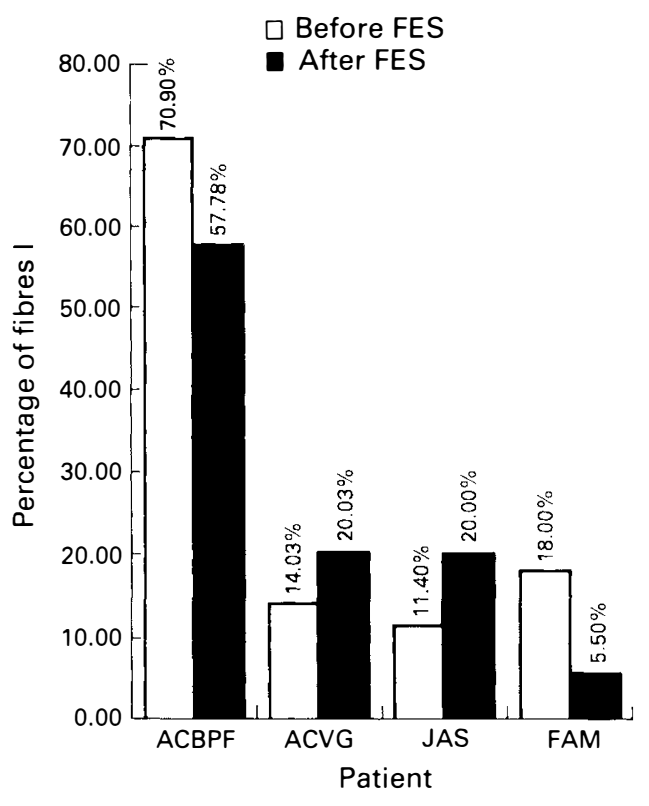

Figure 1 Type I fibres.

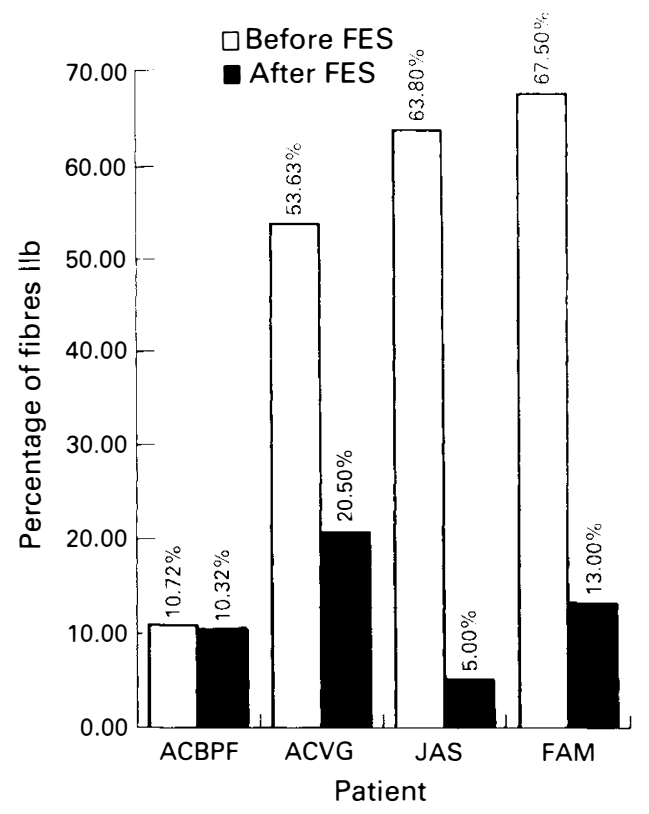

Figure 2 Type IIb fibres.

The initial and final biopsies of cases 1 (Figs 4,5 ) and 2 (Figs 6, 7) are shown; we call attention to the outstanding number of type IIa fibres in the second biopsy. 


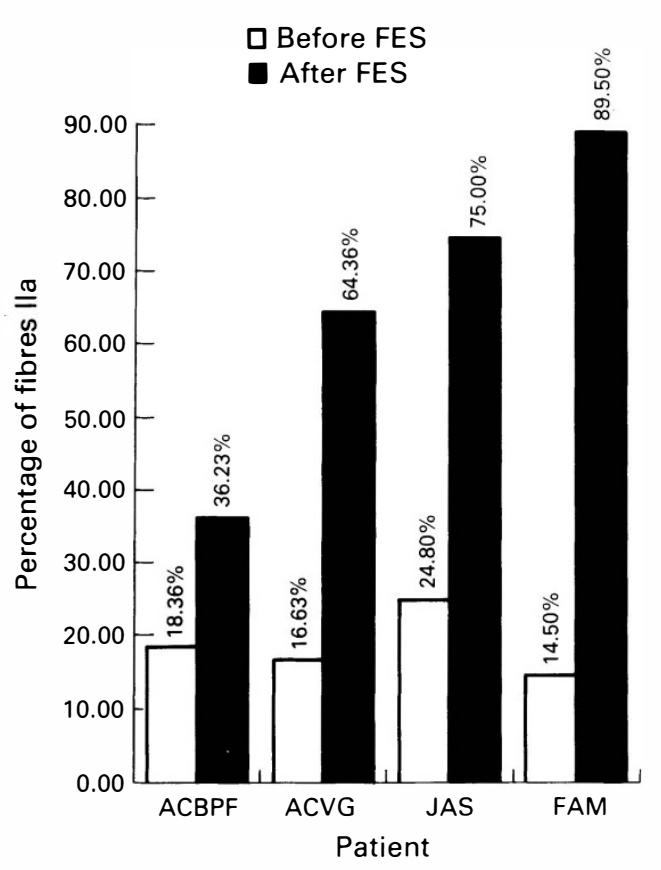

Figure 3 Type IIa fibres.

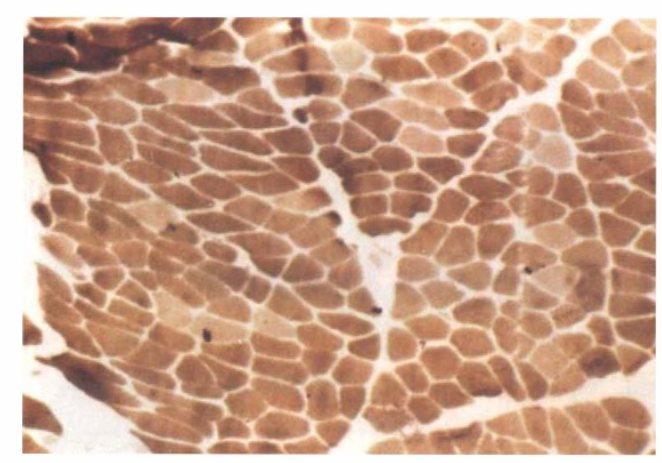

Figure 4 Patient 1. Biopsy before FES.

\section{Discussion}

The initial histochemical muscular pattern of the cases studied showed a high degree of atrophy in all the types of muscle fibres. The normal diameters of muscle fibres in human male adults vary from $40-50$ micras. ${ }^{2}$ Our patients had average diameters less than 30 micras in all types of muscle fibres. All the patients had a spinal cord lesion more than a year previously, which accounts for the

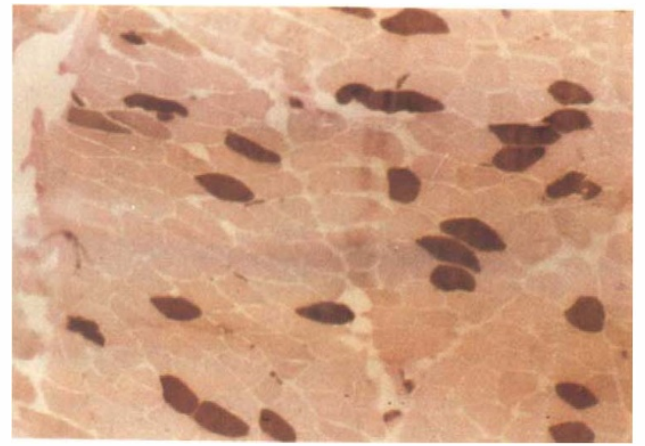

Figure 5 Patient 1. Biopsy after FES.

hypotrophy observed. Reflex activity and the rehabilitation programme were not sufficient to maintain muscle trophism. ${ }^{4}$

The use of FES during the period of 3 months was not able to diminish the initial hypotrophy. The maintenance of the hypotrophy was probably due to the stimulation time: 90 days. This time was not sufficient to improve muscle trophism. A daily training time of 1 hour only was also considered one of the factors that failed to permit muscle hypertrophy. The maintenance of the hypotrophy in our cases, after the stimulation period, could also be attributed to the use of superficial electrodes, since Peckham et $a l^{5}$ obtained improvement in muscle trophism with 25 weeks of training and by using implantation electrodes. Another fact to be considered is the great deal of initial hypotrophy and the difficulty encountered in preventing this pattern in paralysed muscles.

No linear correlation was observed between the different degrees of functional gain because the four patients succeeded in maintaining orthostasis for periods varying from 5 to 15 minutes and two of the patients managed reciprocal locomotion even with the maintenance of the histochemical patterns of hypotrophy. The functional gains obtained through FES are not related to improvement in muscle trophism but are probably due to metabolic changes.

Normal muscle is constituted of slow contraction oxidative fibres (type'I) and type II rapid contraction glycolitic fibres distributed according to genetic inheritance, 


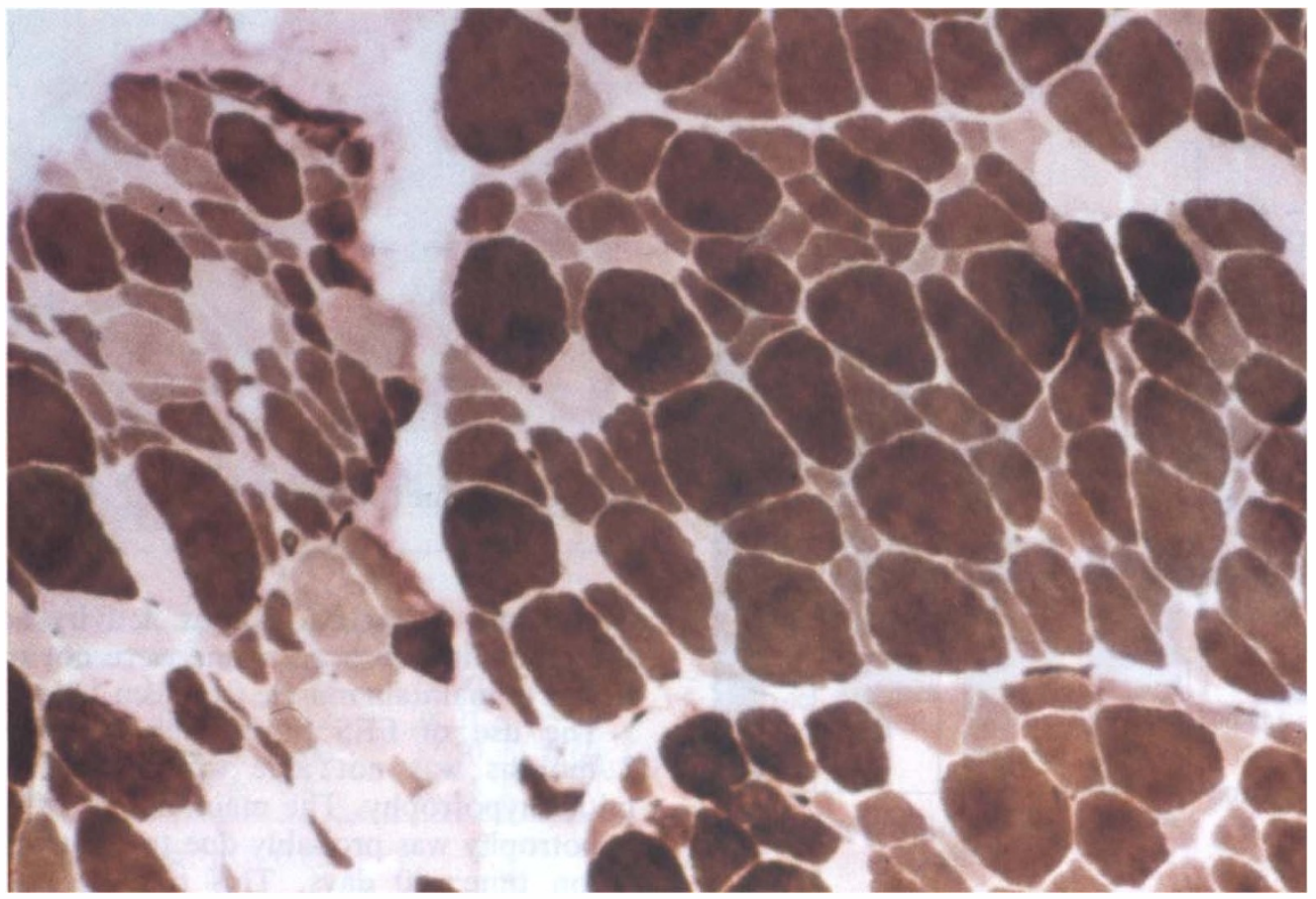

Figure 6 Patient 2. Biopsy before FES.

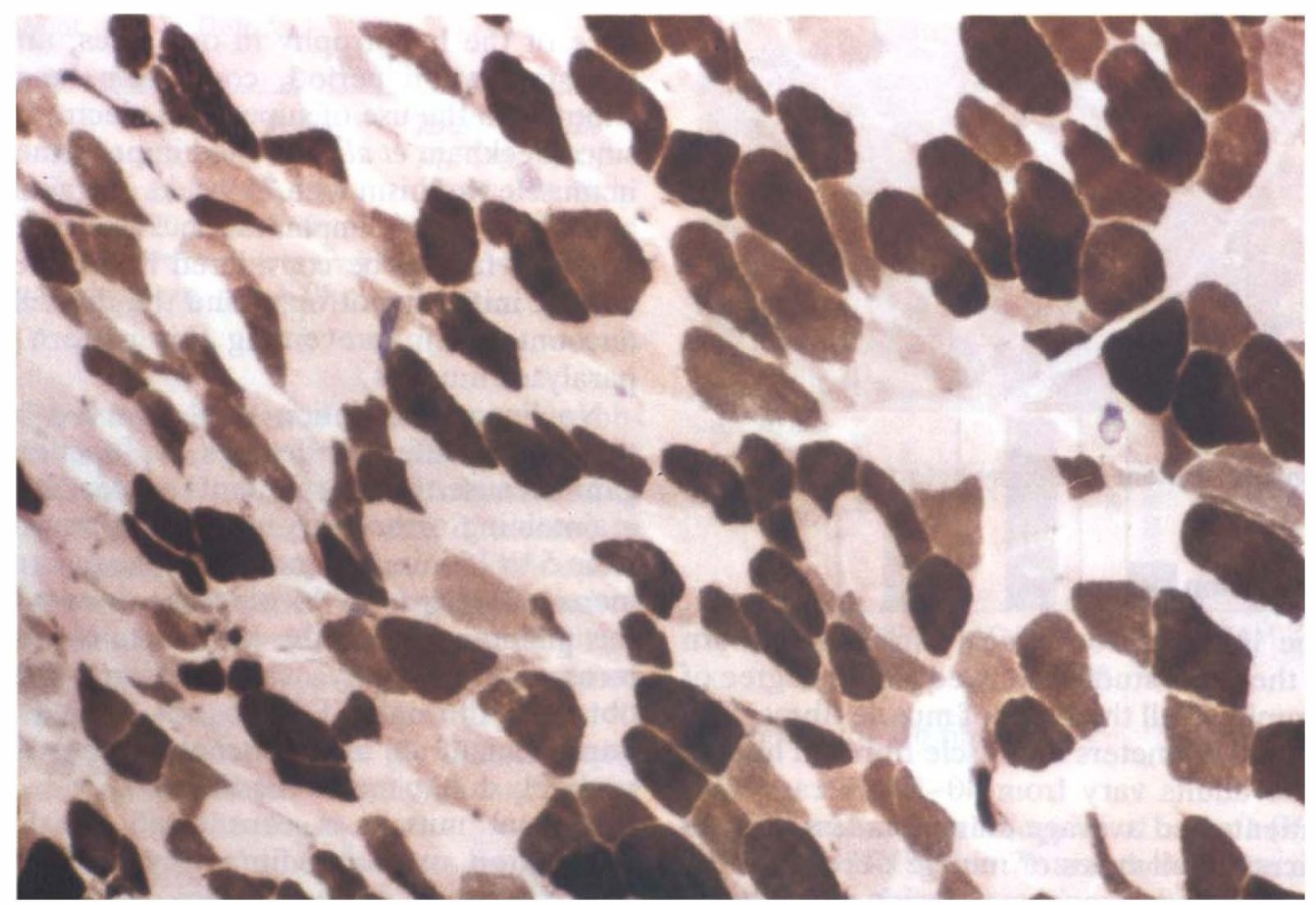

Figure 7 Patient 2. Biopsy after FES. 
the characteristics of the muscles' predominant activity and the type of physical activity. ${ }^{6,7}$

In the initial samples, type IIb fibres predominated in three of the four cases studied and in only one case, type I fibres predominated. Edstrom ${ }^{8}$ relates predominance of type I fibres in the histochemical pattern of Parkinson's disease patients. Mayer et $a l^{9}$ demonstrated in an experimental study, using cats with high thoracic spinal lesions of long term evolution, that there is histological and physiological evidence of a decrease in the number of rapid contraction glycolitic fibres (type IIa) and in slow contraction oxidative fibres (type I), whilst intermediate rapid contraction fibres (type IIb) increase. The altered pattern of muscle activity (spasticity) and the paralysis (disuse) due to spinal cord transection possibly convert slow contraction units into more fatiguable ones. The initial histochemical pattern of three of our cases was consistent with Mayer's findings. ${ }^{9}$

Young et $a l^{10}$ reported that type I slow contraction fibres of the first interosseus muscle of spastic hemiplegic patients were modified to a fourth type of fibre, showing slow contraction and fatiguability. Young et $a l^{11}$ reported that the dynamic and physiological properties of muscles are affected by abnormal innervation patterns, which explains the lower fatiguability threshold in spastic muscles.

In our investigation, the type of current, the frequency used and the surface electrodes applied led to a contraction of 'isometric' type of great force and short duration, in synchronic activity with a great quantity of motor units. This kind of phasic neural activity favours the development of the type II fibres, of rapid contraction and glycolitic metabolism. ${ }^{2}$ This was the result encountered in our samples.

Brindley et al $^{12}$ reported that cyclic training alternating surface and implanted electrodes on the three branches of the femoral nerve, increased resistance to fatigue, although the stimulus was of phasic type. Selective stimulation of motor units simulates neuromuscular activity patterns similar to physiological ones, which explains the greater resistance to fatigue.
The functional results achieved by our patients agree with the histochemical findings: functional improvement, but with activities of short duration and low resistance to fatigue.

Golnick et al $^{13}$ studied needle biopsies of the inferior and superior limb muscles of trained men, using histochemical analysis. The samples taken from trained individuals in aerobic activity (swimming and running) showed a high activity of type I fibres $(84 \%)$. In the individuals trained with anaerobic activities, as weight-lifters, hypertrophy of type II fibres was predominant.

Golnick et $a l^{14}$ in another study reported that histochemical modifications occurred in individuals trained under aerobic circumstances $(75-90 \%$ of maximal capacity). These changes were an increase in the proportion of slow contraction fibres in relation to the ones of rapid contraction. Munsat et $a l^{15}$ observed in experimental studies that the use of FES can convert type II to type I fibres. This finding was not confirmed in our study. Ragnarsson ${ }^{4}$ reported that training with an ergometer with computerised FES for inferior limbs, improves in effective fashion the force and resistance of trained muscles, improving performance, reducing the velocity of contraction and leading to hypertrophy (measured by tomography). Petrofsky et $a l^{16}$ reported improvement in the force and the resistance of the quadriceps with the use of FES, evaluated by clinical criteria. Peckham $e t a l^{5}$ reported that the chronic use of FES leads to muscular hypertrophy and better performance in the individuals tested; these improvements are related to changes in the contractile properties of the trained muscle, but are not related specifically to histochemical changes.

FES probably leads to modifications in the histochemical pattern of the muscle through convertion of type IIb (intermediate) fibres to type IIa (glycolitic) ones. Possibly these metabolic alterations are responsible for the functional gains obtained by the patients, because the fibres are more adequate for orthostasis and locomotion.

In our samples we did not observe the slow contraction high fatiguability intermediate fibres described by Young, ${ }^{10}$ but 
rather the prevalence of intermediate fibres IIB in three of the four cases, which may be due to impaired neural function, as a consequence of the spinal cord lesion: spasticity and immobilisation.

The changes in the histochemical pattern induced by FES, increasing type IIa fibres, is not ideal physiologically for the functions of orthostasis and locomotion of the thoracic paraplegic patient. Our prospects for the future are the use of implanted electrodes and lower frequency currents, which could lead to more physiological changes in muscle patterns.

\section{Conclusions}

The use of FES during 90 days for 30 minutes twice daily did not increase muscle bulk. It modified the pattern of the histochemical analysis of the muscle fibres in SCI patients. This modification was characterised by an increase in the number of type IIa fibres.

\section{References}

1 Gracanin F (1984) Estimulação elétrica funcional. In: Krusen, editor. Tratado de Medicina Física e Reabilitação. 3rd ed. Manole, São Paulo: 381.

2 Riley DA, Allin EF (1973) The effects of inactivity, programed stimulation and denervation on the histochemistry of skeletal muscle fibers types. Exp Neurol 40: 391-413.

3 Niles NR, Chayien J, Cunnigham, Bitensky L (1964) The histochemical demonstration of adenosine triphosphatase activity in myocardium. J Histochem Cytochem 12: 740-743.

4 Ragnarsson KT (1988) Physiologic effects of functional electrical stimulation-induced-exercises in spinal cord-injured patients. Clin Orthop 233: 53-63.

5 Peckham PH, Mortimer JT, Marsolais EB (1976) Alteration in the force and fatiguability of skeletal muscle in quadriplegic humans following exercise induced by chronic electrical stimulation. Clin Orthop 114: 326-334.

6 Wolf SL (1980) Bases funcionais e morfológicas para exercícios terapêuticos. In: Basmajian JV, editor. Terapêutica por Exercícios. 3rd ed. Manole, São Paulo: 57.

7 Lateur BJ, Lehmann JF (1986) Strengthening exercise. In: Leek JC, Gershwin ME, Fowler Jr WM, editors. Principles of Physical Medicine and Rehabilitation in the Musculoskeletal Diseases, 1st ed. Grune \& Stratton, Orlando: 25.

8 Edstrom L (1970) Selective changes in the size of red and white muscle fibres in upper motor neurone lesions and parkinsonism. $1970 \mathrm{~J}$ Neurol Sci 11: 537-541.

9 Mayer RF, Burke RE, Toop J, Walmsley B, Hodgson JA (1984) The effect of spinal cord transection on motor units in cat medial gastrocnemius muscle. Muscle Nerve 7:23-30.

10 Young RR, Mayer RF (1982) Physiological alterations of motor units in hemiplegia. J Neurol Sci 54: 401-410.

11 Young R, Wiegner AW (1986) Spasticity. Clin Orthop 219: 50-62.

12 Brindley GS, Polkey CE, Rushton DN (1979) Electrical splinting of the knee in paraplegia. Paraplegia 16: 428-435.

13 Golnick PD, Armstrong RB, Saubert CW (1972) Enzyme activity and fiber composition in skeletal muscle of untrained and trained men. J Appl Physiol 33: 312-319.

14 Golnick PD, Armstrong RB, Saltin B (1973) Effect of training on enzyme activity and fiber composition of human skeletal muscle. J Appl Physiol 34: 107-111.

15 Munsat TL, Mcneal DN, Waters R (1976) Effects of nerve stimulation on human muscle. Arch Neurol 33: 306-14.

16 Petrofsky JS, Philips CA (1984) The use of functional electrical stimulation for rehabilitation of spinal cord injured patients. CNS Trauma 1: 29-46. 